\title{
Jet Sideways Expansion Effect on Estimating the Gamma-Ray Burst Efficiency
}

\author{
Xiaohong Zhao ${ }^{1,2}$ \\ J. M. Bai ${ }^{1}$
}

\begin{abstract}
The high efficiency of converting kinetic energy into gamma-rays estimated with late-time afterglows in Gamma-Ray Burst (GRB) phenomenon challenges the commonly accepted internal-shock model. However, the efficiency is still highly uncertain because it is sensitive to many effects. In this Letter we study the sideways expansion effect of jets on estimating the efficiency. We find that this effect is considerable, reducing the efficiency by a factor of $\sim 0.5$ for typical parameters, when the afterglow data $\sim 10 \mathrm{hr}$ after the GRB trigger are used to derive the kinetic energy. For a more dense circumburst medium, this effect is more significant. As samples, taking this effect into account, we specifically calculate the efficiency of two bursts whose parameters were well constrained. Almost the same results are derived. This suggests that the sideways expansion effect should be considered when the GRB efficiency is estimated with the late afterglow data.
\end{abstract}

Subject headings: gamma ray: bursts — gamma ray: observations

\section{Introduction}

The efficiency $\left(\eta_{\gamma}\right)$ of converting kinetic energy into $\gamma$-rays $\left(E_{\gamma}\right)$ is one of the most important physical parameters of gamma-ray burst (GRB) phenomenon (Zhang et al. 2007, hereafter Z07). In the conventional internal+external shock GRB model (Rees \& Mészáros 1994; Kobayashi, Piran \& Sari 1997; see recent review by Zhang \& Mészáros 2004), $\eta_{\gamma}$ is 1\%-5\% (e.g., Kobayashi, Piran \& Sari 1997; Kumar 1999), and even under some extreme

\footnotetext{
${ }^{1}$ National Astronomical Observatories/Yunnan Observatory, Chinese Academy of Sciences, P.O. Box 110, 650011 Kunming, China; zhaoxh@vip.sohu.com.

${ }^{2}$ Graduate School of the Chinese Academy of Sciences, 100012, Beijing, China.
} 
assumptions, such as the extremely inhomogeneous velocity of the ejecta shells, $\eta_{\gamma}$ is only 40\% (Kobayashi, Piran \& Sari 1997, Kobayashi \& Sari 2001). However, the $\eta_{\gamma}$ estimated with the late afterglow (say, $10 \mathrm{hr}$ after the GRB trigger) are much higher $(>50 \%)$ than the model prediction (Lloyd-Ronning \& Zhang 2004, hereafter LZ04). By considering the inverse Compton effect, Fan \& Piran (2006, hereafter FP06) derived $\eta_{\gamma}=1 \% \sim 89 \%$. More recently, Z07 revisited this issue for 32 GRBs detected by Swift with the early X-ray afterglow data. They derived the kinetic energy (isotropic-equivalent) at the end of the shallow decay phase $\left(t_{b}\right)$ and at the deceleration time of the fireball $\left(t_{d e c}\right)$, and they found that $\eta_{\gamma}$ estimated with the kinetic energy at $t_{b}$ is $<10 \%$ for most of the GRBs and that the $\eta_{\gamma}$ estimated with the kinetic energy at $t_{d e c}$ varies from a few percent to $>90 \%$. These results challenge the conventional internal-shock model.

The difficulty of measuring $\eta_{\gamma}$ is the derivation of the kinetic energy $\left(E_{k}\right)$ of the afterglows, which sensitively depends on many physical parameters of the burst itself and its environment (Z07). Measurement of the $E_{k}$ with early afterglow data seems more reliable (Z07). However, it is difficult to determine the onset of afterglow from the observation. Furthermore, most of the X-ray early afterglows detected by Swift show complex features with irregular flares (Zhang et al. 2006; Nousek et al. 2006; Panaitescu et al. 2006; Granot et al. 2006). Estimating the kinetic energy with them can also introduce some uncertainties. Zhang et al. (2007) systematically analyzed the prompt and early afterglow emission of 32 Swift bursts and found that the GRB efficiency derived with early afterglow is highly variable. This result - if not intrinsic - seems to prove the above viewpoint. The derivation of $E_{k}$ with the late afterglow data, on the other hand, involves some uncertainties due to the energy loss, energy injection, and jet sideways expansion effect. The advantage of the late afterglow is that this phase is steady, generally showing a smooth power-law decay. By taking the energy loss, the injection, and the jet expansion effect into account, the late afterglow may also be used for estimating $\eta_{\gamma}$.

It is now believed that GRBs arise from jets collimated into a small angle. To derive the actual radiative and kinetic energy from the observed fluence, the collimation correction has to be considered. In previous works, the isotropic-equivalent $E_{\gamma}$ and $E_{k}$ were used to derive the efficiency without considering this geometrical correction, which is only valid when the effect of the jet expanding sideways is ignored. However, the GRB jet evolves and expands sideways with time. Although this effect is not notable at a very early stage (Huang et al. 2000 ), it may affect the estimation of the efficiency $\eta_{\gamma}$, when one uses late afterglows at $\sim 10$ hr to calculate the efficiency. In this Letter, we investigate the jet sideways expansion effect on estimating the GRB efficiency. Our theoretical analysis is present in $\S 2$. Cases studies for some typical GRBs are present in $\S 3$. Discussion and conclusion are presented in $\S 4$. 


\section{Theoretical Analysis}

The $\eta_{\gamma}$ is defined as

$$
\eta_{\gamma} \equiv \frac{E_{\gamma}}{E_{k}+E_{\gamma}}
$$

Assuming $f_{b, \gamma}$ and $f_{b, k}$ are the beaming factors during the prompt and the afterglow phases, respectively, the corrected efficiency $\eta_{\gamma}^{c}$ is given by

$$
\eta_{\gamma}^{c}=\frac{E_{\gamma} f_{b, \gamma}}{E_{k} f_{b, k}+E_{\gamma} f_{b, \gamma}}=\frac{\eta_{\gamma}}{f+(1-f) \eta_{\gamma}}
$$

where $f=f_{b, k} / f_{b, \gamma}$. It can be seen that when jet angle evolution is considered, it is equivalent to increasing the $E_{k}$ by a factor of $f$. For a double-sided jet, $f_{b, \gamma}=1-\cos \left(\theta_{\gamma}\right)$, and $f_{b, k}=1-\cos \left(\theta_{k}\right)$, where $\theta_{\gamma}$ and $\theta_{k}$ are the opening angles during the prompt and the afterglow phases, respectively.

The hydrodynamic evolution of the jet has been studied by a number of authors (e.g., Rhoads 1997, 1999; Panaitescu \& Mészáros 1999; Sari, Piran, \& Halpern 1999; Moderski, Sikora, \& Bulik 2000; Huang et al. 2000; Kumar \& Panaitescu 2000). We adopt the evolution equation group presented in Huang et al. (2000), which describes the overall evolution of relativistic GRB ejecta from the an ultra-relativistic phase to a non-relativistic phase. The equations are quoted as follows:

$$
\begin{gathered}
\frac{d R}{d t}=\beta c \gamma\left(\gamma+\sqrt{\gamma^{2}-1}\right), \\
\frac{d m}{d t}=2 \pi R^{2} \beta c \gamma\left(\gamma+\sqrt{\gamma^{2}-1}\right)(1-\cos \theta) n m_{p}, \\
\frac{d \theta}{d t}=\frac{c_{s}\left(\gamma+\sqrt{\gamma^{2}-1}\right)}{R}, \\
\frac{d \gamma}{d t}=-\frac{2 \pi R^{2} \beta c \gamma\left(\gamma+\sqrt{\gamma^{2}-1}\right)\left(\gamma^{2}-1\right)(1-\cos \theta) n m_{p}}{M_{e j}+\epsilon m+2(1-\epsilon) \gamma m},
\end{gathered}
$$

where $R$ is the radial coordinate in the burst source frame, $\gamma$ is the bulk Lorentz factor of the ejecta $\left[\beta=\left(\gamma^{2}-1\right)^{1 / 2} / \gamma\right], m$ is the swept-up mass, $n$ is the number density of the surrounding interstellar medium (ISM), $m_{p}$ is the mass of the proton, $\theta$ is the half-opening angle of the jet, $c_{s}=\left\{[(\hat{\gamma}(\hat{\gamma}-1)(\gamma-1))]^{1 / 2} /[1+\hat{\gamma}(\gamma-1)]^{1 / 2}\right\} c$ is the comoving sound speed, $\hat{\gamma}=(4 \gamma+1) /(3 \gamma)$ is the adiabatic index, and $\epsilon$ is the radiation efficiency $11 M_{e j}$ is the ejecta

\footnotetext{
${ }^{1}$ This efficiency is the one during the deceleration phase of ejecta after burst which is different from the GRB efficiency
} 
mass that is defined by $E_{i s o}\left(1-\cos \theta_{0}\right)=\gamma_{0} M_{e j} c^{2}$, where $E_{i s o}, \theta_{0}$, and $\gamma_{0}$ are the burst energy (isotropic- equivalent), the initial half-opening angle of jet, and the initial Lorentz factor, respectively.

We first adopt typical parameters as follows in order to study the jet sideways effect: $\gamma_{0}=300, E_{i s o}=10^{53} \mathrm{ergs}, \theta_{0}=0.1$ radians, and $n=1 \mathrm{~cm}^{-3}$. In our calculation, we take $\theta_{\gamma}$ to be the initial half-opening angle of the jet $\theta_{0}$, and $\theta_{k}$ as the one at $10 \mathrm{hr}$. With these typical parameters, we derive the evolution of the jet opening angle, which is shown in Figure 1 . We find that $\theta_{k} \sim 0.14$ at $\sim 10 \mathrm{hr}$. Therefore, $f \sim 2$, and hence $\eta_{\gamma}^{c}=\eta_{\gamma} /\left(2-\eta_{\gamma}\right)$. This indicates that the lower the efficiency, the more significant the reduction is on the efficiency from this effect. For a burst with $\eta_{\gamma}=50 \%, \eta_{\gamma}^{c}=2 \eta_{\gamma} / 3$, while for $\eta_{\gamma}=10 \%, \eta_{\gamma}^{c}=\eta_{\gamma} / 2$. The initial parameters, $E_{i s o}$ and $n$, vary between bursts. Figure 1 also shows the evolution of the jet opening angle for various values of $E_{i s o}$ and $n ; \eta_{\gamma}^{c}$ as a function of the initial parameters is shown in Figure 2. It is found that the correction to $\eta_{\gamma}$ tends to be more significant for a burst with smaller $\theta_{0}$, lower $E_{i s o}$, and a denser ambient medium.

\section{Case Studies}

We take two pre-Swift bursts, GRB 980703 and GRB 000926, as examples. The microphysics parameters of the two bursts have been well constrained with high-quality broadband afterglow data (Yost et al 2003). For calculating a more accurate hydrodynamic evolution, the radiative loss during the early afterglow phase cannot be neglected. Since the energy of the accelerated electrons behind the blast wave is lost both through the synchrotron radiation (inverse Compton cooling is neglected) and through the expansion of the fireball, the radiative efficiency is the ratio of the synchrotron power to the total power, namely (Dai \& Lu 1998,1999b)

$$
\epsilon=\epsilon_{e} \frac{t_{s y n}^{\prime-1}}{t_{s y n}^{\prime-1}+t_{e x}^{\prime-1}},
$$

where $\epsilon_{e}$ is the fraction of shock energy given to the electrons, $t_{s y n}^{\prime}=6 \pi m_{e} c /\left(\sigma_{T} B^{\prime 2} \gamma_{e, \min }\right)$ is the synchrotron cooling time, with $\sigma_{T}$ the Thompson cross section and $\gamma_{e, \text { min }}=\epsilon_{e}(\gamma-$ 1) $m_{p}(p-2) / m_{e}(p-1)+1$ the minimum Lorentz factor of the random motion of electrons in the comoving frame, and $t_{e x}^{\prime}=R /(\gamma c)$ is the comoving frame expansion time. With the numerical factor derived by FP06, we derive

$$
E_{k}=10^{53} \operatorname{ergs} A(p) R_{l} L_{X, 46}^{4 / p+2}\left(\frac{1+z}{2}\right)^{(2-p) /(p+2)} \epsilon_{B,-2}^{-(p-2) /(p+2)} \epsilon_{e,-1}^{4(1-p) /(p+2)},
$$




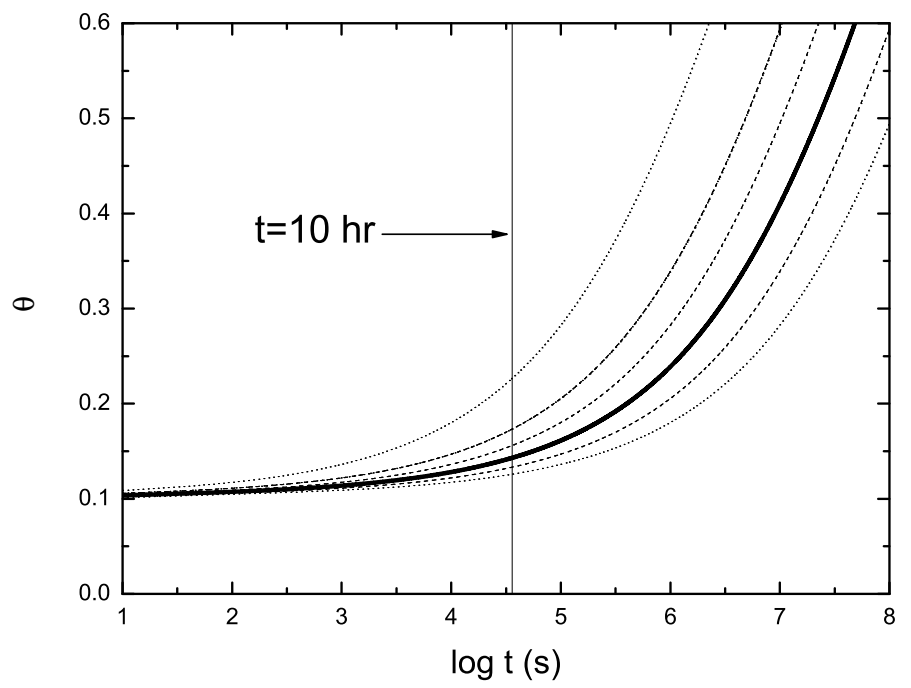

Fig. 1. - Evolution of the jet half-opening angle. The vertical solid line marks the position of $t=10 \mathrm{hr}$. The thick solid line is for the typical parameters. The dotted lines are for the typical parameters with $n=10,000,100$, and $0.01 \mathrm{~cm}^{-3}$ from the top to the bottom. The dashed lines are for the typical parameters with $E_{\text {iso }}=10^{51}, 10^{52}$, and $10^{54}$ ergs from the top to the bottom. Note that the lines for $n=100 \mathrm{~cm}^{-3}$ and $E_{i s o}=10^{51}$ ergs are almost superposed.

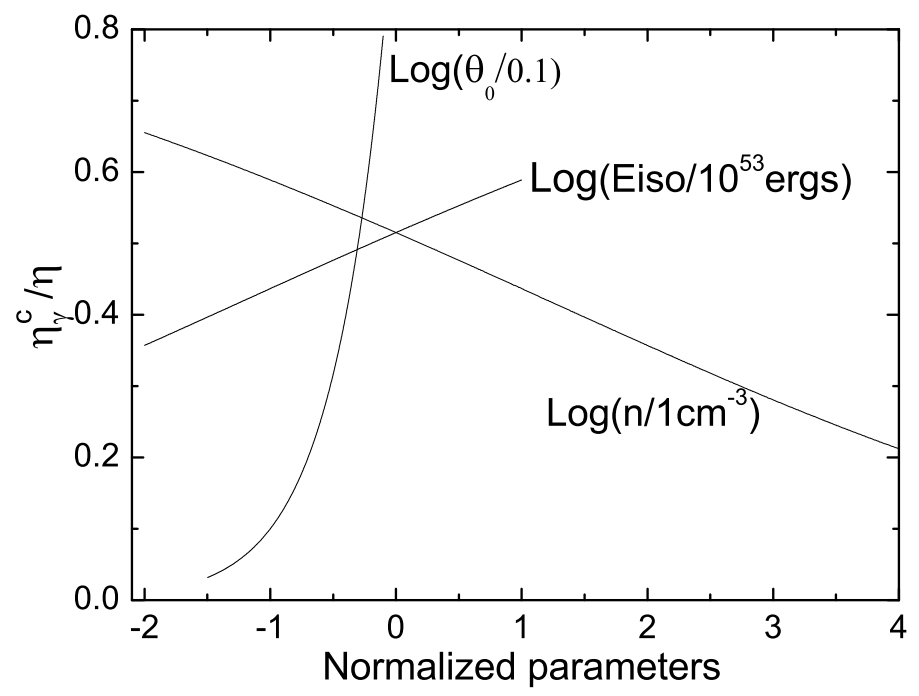

Fig. 2.- The $\eta_{\gamma}^{c}$ in some extent of the initial parameters, which is normalized to $\eta=0.1$ and the initial parameters to the typical values. When one parameter varies, the others are taken as the typical values. 
where

$A(p)=\left\{\frac{1.35 \times 10^{6} \times(1-p / 2)}{0.42^{(2-3 p) / 4}\left(7.6 \times 10^{11}\right)^{(p-1) / 2} C_{p}^{(p-1)}\left[\left(2.42 \times 10^{18}\right)^{(1-p / 2)}-\left(4.84 \times 10^{16}\right)^{(1-p / 2)}\right]}\right\}^{\frac{4}{(p+2)}}$.

Here $p$ is the spectral index of the electron distribution, and $C_{p}=13(p-2) / 3(p-1)$, $R_{l} \sim\left[t(10 h) / T_{90}\right]^{17 \epsilon_{e} / 16}$; is a factor that accounts for radiative losses during the first 10 hr following the prompt phase (Sari 1997, LZ04), $L_{X, 46}=L_{X} / 10^{46}$ is the isotropic X-ray afterglow luminosity at $10 \mathrm{hr}$ (using the value given by Berger et al. 2003), and $\epsilon_{B}$ is the fraction of shock energy given to the magnetic field (the inverse Compton effect is neglected). $E_{k}$ is the isotropic kinetic energy at $t=10 \mathrm{hr}$.

Assuming the jet opening angle at 10 hour is $\theta_{10}$, the initial kinetic energy of ejecta is $E_{0}=E_{k}\left(1-\cos \theta_{10}\right)$. We thus obtain a boundary condition that when $t=t_{10}, \theta=\theta_{10}$. In addition, in the standard model, there is a light-curve break occurring at the time $\left(t_{j}\right)$ when the bulk Lorentz factor of the shock has slowed to $\gamma \sim \theta^{-1}$ (Rhoads 1997; Sari et al. 1999). This can be taken as another boundary condition; namely, when $t=t_{j}, \gamma=\theta^{-1}$. Note that the second condition may depend on the assumed initial Lorentz factor. However, we find that the calculated evolutions of the Lorentz factor with different assumed initial values (from 100 to 500) are almost the same during the late time ( $\gtrsim 1000 \mathrm{~s}$ with the typical parameters), which is understandable. At the late time, the mass of the jet is dominated by the swept-up mass, so the initial mass of ejecta, which is defined by the initial Lorentz factor with a given initial kinetic energy, weakly affects the evolution of the Lorentz factor of a jet. We adjust $\theta_{10}$ and $\theta_{0}$ in a proper range until the above two boundary conditions are satisfied. Then the initial jet angle and the initial kinetic energy are derived, and the efficiency can be calculated. Our results are listed in Table 1. It can be seen that the corrected efficiency is significantly lower than that derived by LZ04 and FP06. Note that the $E_{k}$ derived in this analysis is different from LZ04 and FP06 due to the different microphysics parameters used. If we use the kinetic energy that we obtain, the efficiencies of GRB 980703 and GRB 000926 derived with equation (1) are 0.135 and 0.103 , respectively, while with equation (2), they are only 0.079 and 0.052 , respectively.

\section{Discussions and conclusion}

We have investigated the correction on the estimate of $\eta_{\gamma}$ by considering the jet sideways

effect. Our numerical study shows that for a stronger burst, a burst with a narrower jet opening angle, or a burst in a more dense environment (Dai \& Lu 1999a), this effect tends to be more significant. Using the typical parameters, the corrected efficiency may be reduced 
by a factor of $\sim 0.5$ for a GRB with typical parameters. We use two pre-Swift bursts, GRB 980703 and GRB 000926, as detailed case studies, further confirming our numerical analysis results.

Several authors argued that the efficiencies of some Swift bursts with long time X-ray flattening are as high as 75\%- 90\% (Zhang et al. 2006; Nousek et al. 2006; Ioka et al. 2006; Granot et al. 2006) and that the internal-shock model for GRBs meets the challenge of a so-called efficiency crisis (e.g., Ioka et al. 2006). As mentioned in $\S 1$, the estimate of the GRB efficiency is strongly affected by many effects and depends on unobservable physical parameters. As we show in this analysis, the small jet sideways effect may result in a considerable reduction of the efficiency. More recently, Zhang et al. (2007) estimated the kinetic energy with the data of early afterglows, such as the deceleration time (when the blast wave is decelerated). From the end of GRB to the deceleration time, the jet sideways expansion is insignificant, and thus this effect on the estimate of the GRB efficiency can be negligible. However, they found that the derived GRB efficiency is highly variable from a few percent to $>90 \%$ (at the deceleration time). If these results are intrinsic but not caused by a parameter selection effect or by the uncertainties of early afterglows, they may refresh

our understanding of the GRB phenomenon, and they may require an improvement in the conventional shock model.

We thank Y. Z. Fan for helpful discussion and suggestions. We also acknowledge the referee for constructive comments and suggestions. This work is supported by the National Natural Science Foundation of China (grants 10443003 and 10573030) and the Natural Science Foundation of Yunnan (2003 A0025Q).

\section{REFERENCES}

Berger, E., Kulkarni, S. R., \& Frail, D. A. 2003, ApJ, 590, 379

Bloom, J. S., et al. 2003, ApJ, 594, 674

Dai, Z. G., \& Lu, T. 1998, MNRAS, 298, 87

Dai, Z. G., \& Lu, T. 1999a, ApJ, 519, L155

Dai, Z. G., \& Lu, T. 1999b, ApJ, 520, 634

Fan, Y. Z., \& Piran, T. 2006, MNRAS, 369, 197 (astro-ph/0601054)

Granot, J., Königl, A., \& Piran, T. 2006, MNRAS, 370, 1946 (astro-ph/0601056) 
Huang, Y. F., Gou, L. J., Dai, Z. G., \& Lu, T. 2000, ApJ, 543, 90

Ioka, K., Toma, K., Yamazaki, R., \& Nakamura, T., 2006, A\&A, 458, 7 (astro-ph/0511749)

Kobayashi, S., Piran, T., \& Sari, R. 1997, ApJ, 490, 92

Kobayashi, S., \& Sari, R. 2001, ApJ, 551, 93

Kumar, P., 1999, ApJ, 523, L113

Kumar, P., \& Panaitescu, A. 2000, ApJ, 541, L94

Lloyd-Ronning, N. M., \& Zhang, B., 2004, ApJ, 613, 477

Moderski, R., Sikora, M., \& Bulik, T. 2000, ApJ, 529, 151

Nousek, J. A., et al. 2006, ApJ, 642, 389 (astro-ph/0508332)

Panaitescu, A., \& Mészáros, P. 1999, ApJ, 526, 707

Panaitescu, A., Mészáros, P., Gehrels, N., Burrows, D., \& Nousek, J. 2006, MNRAS, 366, 1357 (astro-ph/0508340)

Rees, M. J., \& Mészáros, P. 1994, ApJ, 430, L93

Rhoads, J. 1997, ApJ, 487, L1

Rhoads, J. 1999, ApJ, 525, 737

Sari, R., Piran, T., \& Halpern, J. P. 1999, ApJ, 519, L17

Sari, R. 1997, ApJ, 489, L37

Yost, S. A., Harrison, F. A., Sari, R. \& Frail, D. A. 2003, ApJ, 597, 459

Zhang, B., \& Mészáros, P., 2004, IJMPA, 19, 2385

Zhang, B., et al. 2006, ApJ, 642, 354

Zhang, B., et al. 2007, ApJ, in press (astro-ph/0610177) 
Table 1: The parameters and the calculated GRB efficiency. $E_{\gamma}$ is the isotropic radiative energy from Bloom et al. (2003), microphysics parameters, $n$ and $t_{j}$ from Yost et al. (2003). Here we assume $\gamma_{0}=300$. $a$ and $b$ are the efficiency from LZ04 and FP06, respectively.

\begin{tabular}{|c|c|c|c|c|c|c|c|c|c|}
\hline GRB & $\begin{array}{c}E_{\gamma} \\
\left(10^{52} \mathrm{ergs}\right)\end{array}$ & $\theta_{0}$ & $\epsilon_{e}$ & $\epsilon_{B}$ & $p$ & $\begin{array}{c}\mathrm{E}_{k} \\
\left(10^{53} \mathrm{ergs}\right)\end{array}$ & $\begin{array}{c}n \\
\left(\mathrm{~cm}^{-3}\right)\end{array}$ & $\begin{array}{c}t_{j} \\
(\text { days })\end{array}$ & $\begin{array}{c}\eta_{\gamma}^{c} \\
(\mathrm{a}, \mathrm{b})\end{array}$ \\
\hline 980703 & 6.01 & $8.37^{\circ}$ & 0.27 & 0.0018 & 2.54 & 3.85 & 28 & 3.4 & $\begin{array}{c}0.08 \\
(0.71,0.21)\end{array}$ \\
\hline 000926 & 27.97 & $4.59^{\circ}$ & 0.15 & 0.022 & 2.79 & 24.3 & 16 & 2.6 & 0.05 \\
$(0.74,0.23)$ \\
\hline
\end{tabular}

